Journal of Bangladesh Academy of Sciences, Vol. 33, No. 2, 189-197, 2009

\title{
EFFECTS OF CO-DOPED BIMETALLIC IMPURITIES ON THE GROWTH AND NUCLEATION KINETICS OF POTASSIUM ACID PHTHALATE (KAP)
}

\author{
S. A. BEGUM AND J. PODDER ${ }^{* 1}$ \\ Ideal School and College, Motijheel, Dhaka-1000, Bangladesh
}

(Received revised copy: July 7, 2009)

\begin{abstract}
Single crystals of pure potassium acid phthalate (KAP) and nickel-magnesium $\left(\mathrm{Ni}^{2+}-\mathrm{Mg}^{2+}\right)$, cobalt-magnesium $\left(\mathrm{Co}^{2+}-\mathrm{Mg}^{2+}\right)$ and lithium-magnesium $\left(\mathrm{Li}^{1+}-\mathrm{Mg}^{2+}\right)$ co-doped potassium acid phthalate crystals were grown by an isothermal evaporation method. Good quality and optically transparent single crystals of pure and bimetallic doped KAP were grown. Enhancement of the metastable zonewidth was achieved by the addition of co-doped novel bimetallic impurities in KAP solution. The induction period and various critical nucleation parameters such as radius of critical nucleus, number of molecules in the critical nuclei and the critical free energy change were calculated based on the classical theory for homogeneous nucleation. The induction period decreases exponentially with supersaturation ratio and hence the nucleation rate increases with the increase of supersaturation ratio for all systems. Subsequent incorporation of these bimetallic codoped impurities into the crystal would allow the growth rate to achieve its maximum value. The induction period is found to increase with the incorporation of $\mathrm{Ni}^{2+}, \mathrm{Co}^{2+}$ and $\mathrm{Li}^{1+}$ ions doped along with $\mathrm{Mg}^{2+}$ ions in KAP. It is anticipated that these larger cations play a vital role in the growth mechanism and it favors to reduce the formation of secondary nucleation by positioning the doped metal ions into the interstitial sites.
\end{abstract}

\section{INTRODUCTION}

Potassium acid phthalate (KAP) with chemical formula $\mathrm{K}\left(\mathrm{C}_{6} \mathrm{H}_{4} \mathrm{COOH} . \mathrm{COO}\right)$, is a promising semi-organic crystal which crystallizes into the orthorhombic form with four molecules per unit cell and space group $P a c 2_{1}{ }^{(1)}$. KAP single crystals exhibit excellent physical properties and also piezoelectric, pyroelectric and nonlinear properties ${ }^{(2-4)}$. It is widely used as X-ray monochromator and X-ray analyzer. KAP is extensively studied for its crystal morphology ${ }^{(5)}$. Many studies on the growth kinetics of KAP with different impurities were reported earlier and the changes of the crystal morphology and supersaturation were also discussed ${ }^{(6-8)}$. The addition of some transition metal ions as impurities is expected to influence the growth kinetics, habit modification as well as growing the large size single crystal. The presence of small amount of impurities such as $\mathrm{Ni}^{2+}, \mathrm{Mg}^{2+}, \mathrm{Co}^{2+}$ and $\mathrm{Li}^{1+}$ plays a vital role on the growth rate, habit of the crystal and its properties ${ }^{(9)}$. In order to grow bulk crystals with faster growth rates along all the crystallographic directions; it is necessary to know the solubility, metastable zonewidth, induction period and nucleation parameters such as radius of critical nucleus, critical free

*Corresponding author: ipodder@phy.buet.ac.bd,

${ }^{1}$ Department of Physics, Bangladesh University of Engineering and Technology, Dhaka-1000, Bangladesh 
energy, etc. to optimize the growth conditions. The aim of this work is to grow transparent and good quality KAP crystal by doping it with bimetallic cations (magnesium-nickel, magnesium-cobalt, and magnesium-lithium). In this paper, we present a systematic investigation on the growth kinetics and nucleation parameters, such as solubility, metastable zonewidth, induction period, interfacial energy, critical radius, nucleation rate, etc. by doping with bimetallic impurities for growing bulk KAP crystals.

\section{EXPERIMENTAL}

\section{Solubility}

The solubility of a chemical species in a solvent reflects to the amount of solute to be dissolved completely at constant temperature, pressure and solvent composition. The solubility of KAP in pure state and that of KAP doped with bimetallic impurities were used in the present study. Nickel sulphate, cobalt sulphate, magnesium sulphate, lithium sulphate were used as sources of $\mathrm{Ni}^{2+}, \mathrm{Co}^{2+}, \mathrm{Mg}^{2+}$ and $\mathrm{Li}^{1+}$ cations. Analar grade KAP $\left(\mathrm{C}_{8} \mathrm{H}_{5} \mathrm{O}_{4} \mathrm{~K}\right)$, E-Merck, double distilled water was used in the present study. To grow bulk crystals from solution by isothermal evaporation technique, it is desirable to select a solvent in which it is moderately soluble. The size of a crystal depends on the amount of materials available in the solution, which in turn is decided by the solubility of the material in that solvent. The solubility of KAP in the above solvent was determined by adding solute in solvent maintained at constant temperature till it is completely dissolved. The mother solution was prepared using the solubility relation ${ }^{(10)}$,

$$
\mathrm{C}(\mathrm{T})=9.283-0.059 \mathrm{~T}+0.0058 \mathrm{~T}^{2} \text { of KAP in water }
$$

where $\mathrm{C}(\mathrm{T})$ is the solubility of KAP in water $(\mathrm{g} / 100 \mathrm{ml})$ and $\mathrm{T}$ is the temperature $\left({ }^{\circ} \mathrm{C}\right)$. Stirring was achieved using an immersible teflon coated magnet. The solution was stirred continuously for six hours to achieve stabilization. Using this technique, we have evaluated the magnitude of solubility of KAP for various temperatures $\left(30-50^{\circ} \mathrm{C}\right)$. The solubility curve of pure KAP is shown in Fig. 2.

\section{Metastable zone width}

The saturated solution of KAP, nickel-magnesium doped KAP (NMKAP), cobaltmagnesium doped KAP (CMKAP) and lithium-magnesium doped KAP (LMKAP) were prepared in accordance with the solubility data for the nucleation experiments. The width of metastable zone in aqueous solutions can be measured by an isothermal or polythermal method $^{(11)}$. Hence the metastable zonewidth of above saturated solution was measured by the conventional polythermal method. These studies were carried out in a constant temperature bath (accuracy $\pm 0.1^{\circ} \mathrm{C}$ ). A constant volume of $100 \mathrm{ml}$ solution was used in all the experiments. The saturated solution was preheated to $5^{\circ} \mathrm{C}$ above the saturation temperature for homogenization and the solution was maintained at the super heated temperature for 1 hour before cooling. The solution was continuously stirred using an 
immersible teflon coated magnet to ensure homogeneous concentration. In the polythermal method, the equilibrium saturated solution was cooled from the over heated temperature until the temperature reached at which the first visible critical nucleus was observed. The direct visual method was adopted for studying the nucleation temperature. The difference between the saturated temperature and the nucleation temperature is taken to be the metastable zonewidth of the system. The experiment was repeated for different saturation temperatures, $35,40,45$ and $50^{\circ} \mathrm{C}$. The metastable zonewidth for different saturation temperatures for pure and bimetallic doped solutions is shown in Fig. 2.

\section{Induction period and nucleation kinetics}

The induction period of pure KAP, NMKAP, CMKAP and LMKAP solutions were measured by means of isothermal method ${ }^{(12)}$. The direct visual method was used for measuring induction period. Aqueous solutions of equal volume $(100 \mathrm{ml})$ were taken in the cells at a higher temperature. As the temperature of the cell reached the temperature of the bath at $30^{\circ} \mathrm{C}$, the time was noted. The time required for the growth of critical nucleus to a detectable size is negligibly small compared with the time needed between the attainment of supersaturation and the appearance of nucleus of detectable size and hence this can be taken as induction period. The appearance of first speck of the nucleus was seen at the bottom of the container. Induction periods were recorded for different supersaturation ratios of $1.03,1.05,1.07,1.09$ and 1.11 for pure KAP and various bimetallic doped KAP. Repeated trials were performed to ascertain the correctness of the observed results. The induction period is written as

$$
\tau \propto \exp \left(\frac{\Delta G^{*}}{k T}\right)
$$

where $\Delta \mathrm{G}^{*}$ is the critical free energy change of the nucleus, $\mathrm{k}$ is the Boltzmann constant and $\mathrm{T}$ is the absolute temperature of the solution.

$$
\begin{aligned}
& \ln \tau=\ln B+\left(\frac{\Delta G^{*}}{k T}\right) \\
& \text { and } \Delta G^{*}=\frac{16 \pi \sigma^{3}}{3 \Delta G_{\mathrm{v}}^{2}}
\end{aligned}
$$

where $B$ is a constant, $\sigma$ is the interfacial energy, $\Delta G_{v}$ is the Gibb's free energy per unit volume. The interfacial energy of the inter phase between the growing crystal and the surrounding mother phase is a critical parameter of the nucleation and growth of crystals.

The bulk change of energy per unit volume is given as

$$
\Delta G_{v}=\frac{-k T \ln S}{V}
$$


where $\mathrm{V}$ is the specific volume and is obtained by the expression,

$$
V=\frac{\text { Molecularweight }}{\text { density } \times N_{A}}
$$

and $\mathrm{S}$ is the supersaturation ratio, given by $S=\frac{C}{C_{0}}, \mathrm{C}$ is the actual concentration, $\mathrm{C}_{0}$ is

the equilibrium concentration, $\mathrm{N}_{\mathrm{A}}$ is the Avogadro's number. The interfacial energy has been calculated using the experimentally determined induction period values by the following relation on the basis of classical theory for homogeneous formation of spherical nuclei ${ }^{(13)}$

$$
\ln \tau=\ln B+\frac{16 \pi \sigma^{3} v^{2} N_{A}}{3 R^{3} T^{3}(\ln S)^{2}}
$$

Where $v$ is the molar volume of the crystals, $\mathrm{R}$ the gas constant and $\tau$ the induction period of KAP and bimetallic doped KAP solution at temperature T. A plot of $1 /(\ln S)^{2}$ against $\ln \tau$ is a straight line. The intercept of straight line on the $y$-axis gives the value of $\operatorname{lnB}$. The equation suggests a straight line fit for $\ln \tau$ against $1 /(\ln S)^{2}$ with a slope given by

$$
m=\frac{16 \pi \sigma^{3} v^{2} N_{A}}{3 R^{3} T^{3}}
$$

Since $\ln B$ weakly depends on temperature, the interfacial energy $\sigma$ of the solid relative to the solution has been calculated from the slope of the lines as

$$
\sigma^{3}=\frac{3 R^{3} T^{3} m}{16 \pi v^{2} N_{A}}
$$

where $m$ is the slope of the straight line. Using the interfacial tension value, the radius of critical nucleus $\left(\mathrm{r}^{*}\right)$ and number of molecules in the critical nucleus $\left(\mathrm{i}^{*}\right)$ were calculated.

$$
r^{*}=\frac{2 \sigma v}{R T \ln S}
$$

The number of molecules in the critical nucleus ${ }^{(14)}$ is expressed as

$$
i^{*}=\frac{2 \pi(r *)^{3}}{3 V}
$$

The relationship between rate of nucleation (the number of nuclei formed per unit time per unit volume) and induction period (the time taken between the attainment of supersaturation and the appearance of detectable nucleus) can be expressed as ${ }^{(15)}$

$$
J=\frac{1}{\tau}=A \exp \left(-\frac{\Delta G^{*}}{k T}\right)=A \exp \left(\frac{16 \pi \sigma^{3}}{3 k T \Delta G_{v}^{2}}\right)
$$

Where $\mathrm{A}$ is a pre exponential factor. 


\section{RESULTS AND DISCUSSION}

The bimetallic doped as grown KAP crystals are shown in Fig. 1. The metastable zonewidth of KAP, NMKAP, CMKAP and LMKAP solutions is shown in Fig. 2 comparing with the pure system. It is obvious from Fig. 2 that the zonewidths for all the solutions decrease as the temperature increases. At the same time, addition of different bimetallic dopants enhances the metastable zonewidth of KAP solutions for all the temperatures and makes KAP solution more stable. Also, it is observed that during the experiment, the number of tiny crystals formed by spontaneous nucleation is appreciably reduced in presence of different bimetallic dopants compared to that of pure one. This might be due to the incorporation of bimetallic impurity species into the adsorption layer which might cause reducing the activities of heterogeneous nucleation.
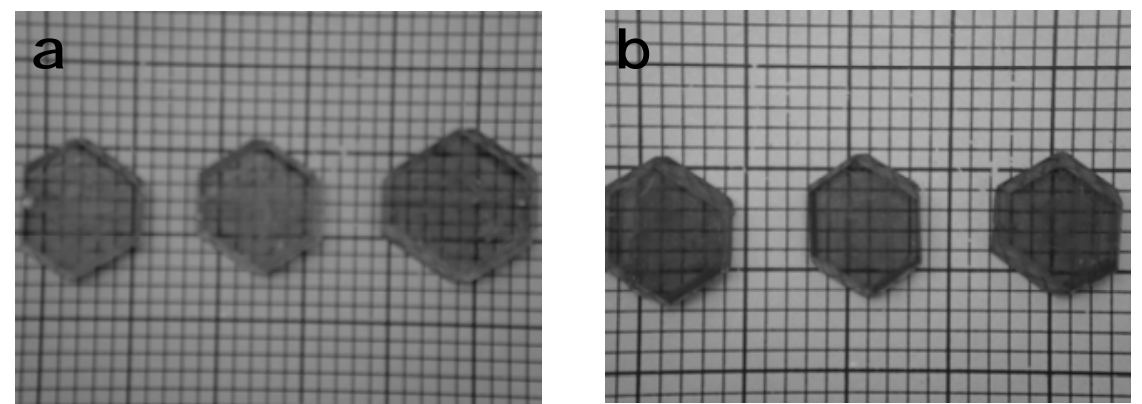

Fig. 1. Photographs of (a) $\mathrm{Ni}^{2+}-\mathrm{Mg}^{2+}$ doped KAP crystals; (b) $\mathrm{Co}^{2+-}-\mathrm{Mg}^{2+}$ doped KAP crystals

The experimental results on induction period $(\tau)$ for the pure and different bimetallic doped KAP crystals are presented in Fig. 3. The plots show that the induction period decreases exponentially with the supersaturation ratio, which suggests that the nucleation rate increases exponentially. It means that the number of critical nuclei formed increases thereby leading to spurious nucleation.

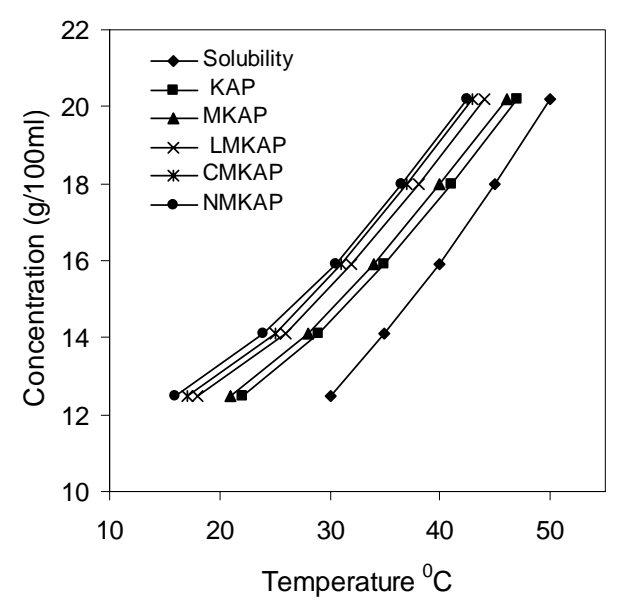

Fig. 2. Solubility and metastability limit curves for pure and different bimetallic doped KAP as a function of temperature. 


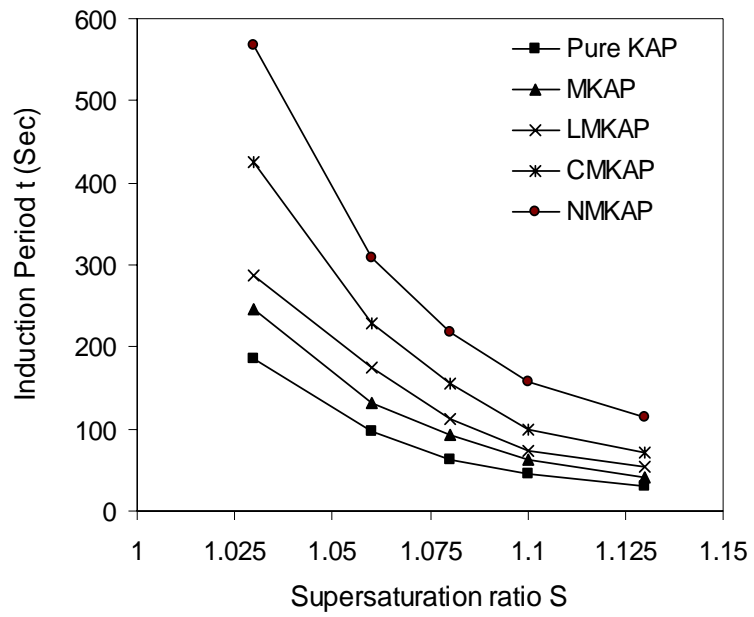

Fig. 3. Induction period vs supersaturation ratio for pure and different bimetallic doped KAP solution.

According to the principles of homogeneous and heterogeneous nucleation theories, the free energy of formation of a nucleus under heterogeneous nucleation is less than that of homogeneous nucleation. Considering the different dopants added system, it can be noticed that the induction period increases with the different dopants of same molar concentration compared to that of pure system. The presence of bimetallic dopants is affecting the nucleation behavior considerably. So it may be inferred that the secondary nucleation can be minimized by the addition of bimetallic impurities like $\mathrm{Ni}^{2+}, \mathrm{Co}^{2+}, \mathrm{Li}^{1+}$ co-doped along with $\mathrm{Mg}^{2+}$ in KAP solutions. Plot of $\ln \tau$ against $1 /(\ln S)^{2}$ is shown in Fig. 4. In order to reduce the effect of heterogeneous nucleation on the nucleation parameters, the results were obtained using the slopes determined by a straight line fit for $\ln \tau$ against $1 /(\ln S)^{2}$.

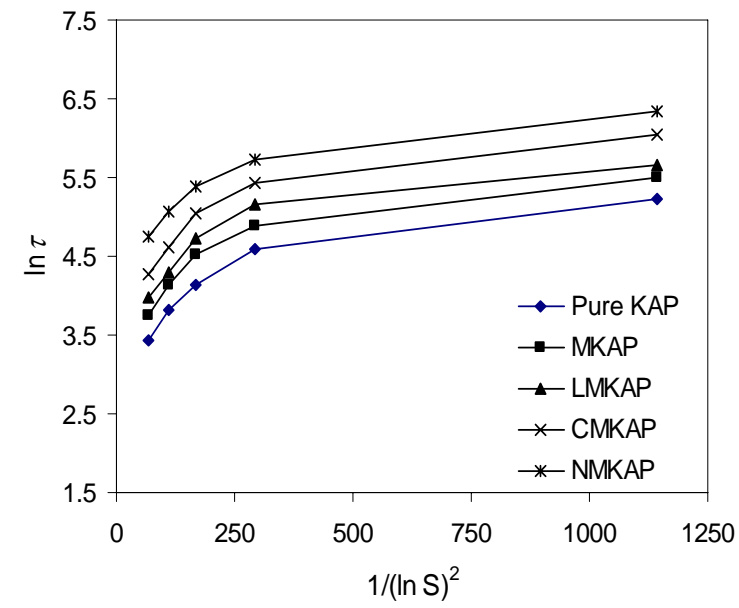

Fig. 4. Plot of $\ln \tau$ vs. $1 /(\ln S)^{2}$ for pure and different bimetallic doped KAP solution. 
The interfacial energy has been calculated for pure KAP and different bimetallic doped KAP crystals. The graph drawn between interfacial energy $\sigma$ and supersaturation ratio $\mathrm{S}$ is shown in Fig. 5. The calculated interfacial energy varies from 1.438 to $1.598 \mathrm{~mJ} / \mathrm{m}^{2}$.

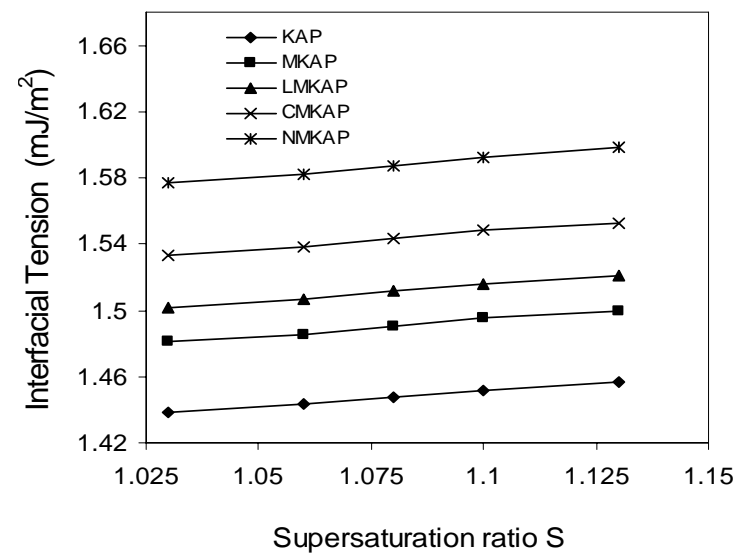

Fig. 5. Interfacial energy vs. supersaturation ratio for pure and different bimetallic doped KAP crystals.

Using the interfacial energy values, the nucleation parameters such as the radius of critical nucleus $\mathrm{r}^{*}$, the free energy for the formation of the critical nucleus $\Delta \mathrm{G}^{*}$ and number of molecules in the critical nucleus $i^{*}$ have been calculated for the controlled nucleation condition. Plot of $r^{*}$ and $\Delta G^{*}$ against supersaturation ratio are shown in Fig. 6 and Fig. 7 respectively. It was observed that the value of $r^{*}, i^{*}$ and $\Delta G^{*}$ decreased with the increase of supersaturation ratio and as a result the nucleation rate $J$ increases with the increase of supersaturation ratio which also varies with different bimetallic dopants shown in Fig. 8 . Hence it is concluded that the interfacial energy plays a vital role in nucleation mechanism. The ionic radii of $\mathrm{Ni}, \mathrm{Co}, \mathrm{Li}$, and $\mathrm{Mg}$ are $0.69 \AA, 0.74 \AA, 0.76 \AA$ and $0.72 \AA$, respectively. Hence the larger interfacial energy resulted in a longer induction period could be due to incorporation of the larger cations $\mathrm{Ni}^{2+}, \mathrm{Co}^{2+}, \mathrm{Li}^{1+}$ co-doped with $\mathrm{Mg}^{2+}$ in $\mathrm{KAP}$ solutions. It might be due to change of inter ionic distances of the particles of solute by the larger size of the doped ions and hence the formation of cluster is getting difficult. Thus these metal ions have great influence on the interfacial energy and the induction period.

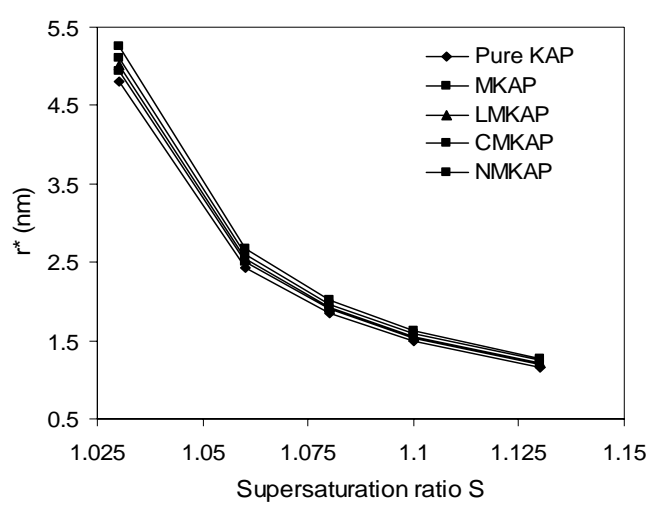

Fig. 6. Critical nucleus vs. supersaturation ratio for pure and different bimetallic doped KAP crystals. 


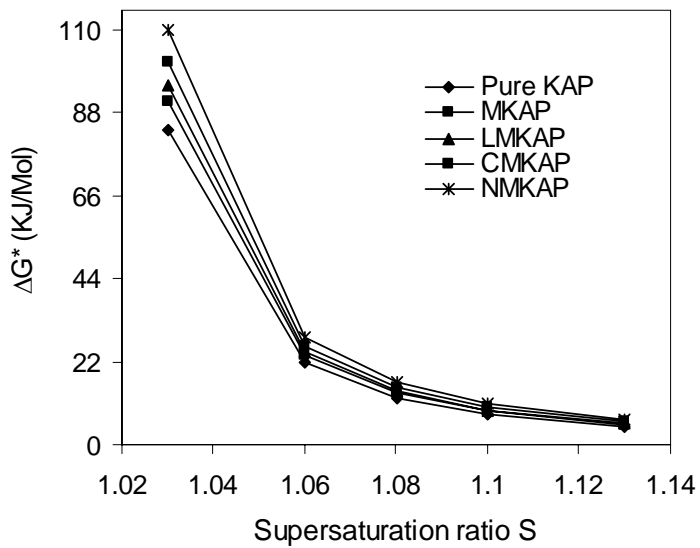

Fig. 7. Critical free energy vs. supersaturation ratio for pure and different bimetallic doped KAP crystals.

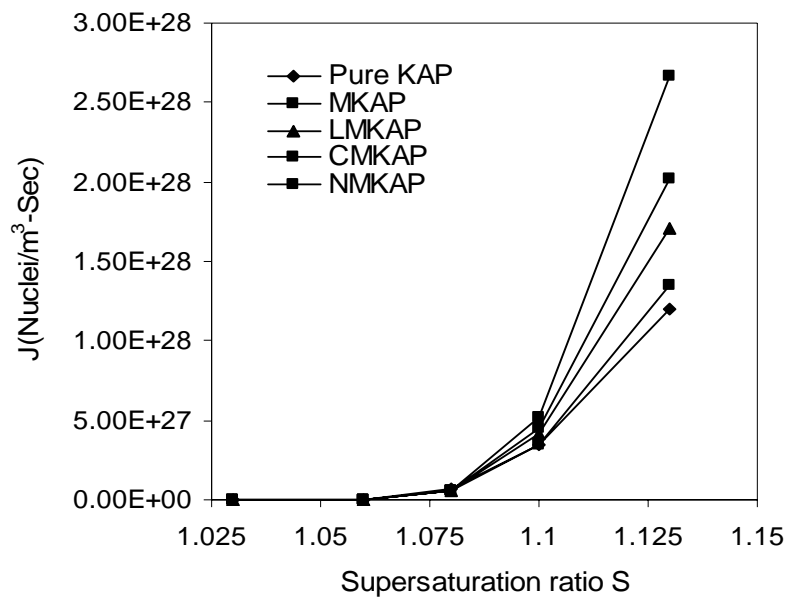

Fig. 8. Nucleation rate vs. supersaturation ratio for pure and different bimetallic doped KAP crystals

\section{CONCLUSIONS}

The fundamental growth parameters have been investigated for pure and different bimetallic doped KAP crystals in supersaturated aqueous solutions. The effects of different bimetallic impurities on KAP solution are found to increase the metastable zonewidth considerably. This may be due to the suppression of chemical activity of the heavy metal ions which are present as impurities in the KAP solutions. The nucleation parameters like induction period, interfacial energy, energy of formation and critical radius of the nucleus are found to increase in presence of co-doped bimetallic impurities. The increase in induction time is due to an increase in the crystal-solution interfacial energy at higher size cations $\mathrm{Ni}^{2+}, \mathrm{Co}^{2+}, \mathrm{Li}^{1+}$ co-doped with $\mathrm{Mg}^{2+}$. It is anticipated that the dopants may substitute or enter into the interstitial sites. Colouration is observed in nickel and cobalt co-doped with magnesium in KAP crystals. This may be due to incorporation 
of larger sized cations like $\mathrm{Ni}^{2+}$ and $\mathrm{Co}^{2+}$ along with $\mathrm{Mg}^{2+}$ into the superficial crystal lattice and forming defect centers. The findings of this study would be useful to achieve good quality bulk KAP crystals from aqueous solutions with faster growth.

\section{REFERENCES}

1. S. K. Geetha, R. Perumal, S. Moorthy Babu, P. M.Anbarasan, Cryst. Res. Technol, 41, 221, 2006.

2. L. Beck, P. Stemmler, AND F. Legrand, Rev. Sci. Inst. 66, 1601, 1995

3. M. A. BARTKIEWICZ, Adv. Mater. Opt. Electron. 2, 157, 1993.

4. K. N. SRinivasan, Opt. Mat. 27, 389, 2004.

5. M. H. J. Hottenhuis, C. B. LuCAsius, J. Cryst. Growth 91, 623, 1989.

6. K. Uthayarani, R. Sankar And C. K. Shashidharan Nair, Cryst. Res. Technol. 43, 733, 2008.

7. R. Mohan Kumar, D. Rajan Babu, P. Murugakoothan, R. Jayavel, J. Cryst. Growth 245, 297, 2002.

8. M. H.J. Hottenhuis AND C.B. LuCAsius, J. Cryst. Growth 78, 379, 1986.

9. V.G. DMitRiev, Hand book of non linear optical crystal, Springer Verlag, Berlin, Heidelberg, pp 5-25, 1991.

10. K. Yamashita, N. Watanable, O. Matsuvo, J. Yamazaki, I. Hatsukade, T. Ishigami, S. TAKahama, K. Tamura, AND M. OHATANi, Rev. Sci. Instr. 63, 1217, 1992.

11. N. P. Rajesh, P. Santhana Raghavan, P. Ramasamy, C. W. Lan, And J. Chin, Inst. Chem. Engrs. 33, 325, 2000.

12. P. V. Dhanaraj, Santheep K. Mathew, And N. P. Rajesh, J. Cryst. Growth 310, 2532, 2008.

13. R. Sankar, C. M. Raghavan, And R. Jayavel, Cryst. Res. Technol. 41, 919, 2006.

14. T. Kanagasekaran, M. Gunasekaran, P. Srinivasan, D. Jayaraman, R. Gopalakrishnan, AND P. Ramasamy, Cryst. Res. Technol. 40, 1128, 2005.

15. O. Sahin, H. Dolas and H. Demir, Cryst. Res. Technol. 42, 766, 2007. 